\title{
Cholesterol and ApoE in Alzheimer's disease
}

\author{
Marie-Claude Potier ${ }^{1, *}$, Linda Hanbouch ${ }^{1}$ and Catherine Marquer ${ }^{2}$ \\ ${ }^{1}$ Institut du Cerveau et de la Moelle épinière, CNRS UMR7225, INSERM U1127, UPMC, Hôpital de la Pitié-Salpêtrière, \\ 47 Bd de l'Hôpital, Paris, France \\ ${ }^{2}$ Taub Institute for Research on Alzheimer's disease and the Aging Brain and Department of Pathology and Cell Biology, \\ Columbia University Medical Center, New York City, NY 10032, USA
}

Received 2 April 2018 - Accepted 15 June 2018

\begin{abstract}
Genetic, neuropathological and biochemical studies suggest strong links between cholesterol, the apolipoprotein E (APOE) and Alzheimer's disease (AD), both in humans and in animal models of the disease. From the literature and our work, we can predict that transient increase of the levels of cholesterol at the membrane of neurons would profoundly affect the processing of the transmembrane Amyloid Precursor Protein (APP) by triggering its clathrin dependent endocytosis and the resulting production of amyloid- $\beta$ $(\mathrm{A} \beta)$ peptides. Here, we will review these data together with structural and molecular dynamic studies that characterized the role of cholesterol on APP conformation and positioning at the membrane. Specifically decreasing brain cholesterol or replacing it with plant sterols crossing the blood brain barrier appear like promising strategies to either delay or counteract the development of sporadic AD.
\end{abstract}

Keywords: Alzheimer's disease / cholesterol / Apo lipoprotein E / endosome / amyloid

Résumé - Cholesterol et ApoE dans la maladie d'Alzheimer. Des études génétiques, neuropathologiques et biochimiques suggèrent des liens étroits entre le cholestérol, l'apolipoprotéine E (APOE) et la maladie d'Alzheimer (MA), chez l'homme et dans des modèles animaux de la maladie. Les études publiées et nos travaux nous permettent de prédire qu'une augmentation transitoire des taux de cholestérol à la membrane des neurones affecterait profondément le clivage de la protéine transmembranaire précurseur de l'amyloïde (APP) en déclenchant son endocytose dépendante de la clathrine et la production résultante de peptides amyloïdes $\beta$. Dans cette revue nous colligeons ces données ainsi que des études de dynamique structurale et moléculaire modélisant le rôle du cholestérol sur la conformation APP et son positionnement à la membrane. La diminution spécifique du cholestérol cérébral ou son remplacement par des phytostérols traversant la barrière hématoencéphalique apparaissent comme des stratégies prometteuses pour retarder ou contrecarrer le développement de la MA sporadique.

Mots clés : maladie d'Alzheimer / cholestérol / Apolipoproteine E / endosome / amyloïde

\section{Introduction}

Lipids are essential for brain function. Brain is one of the tissues with the highest lipid content, with up to $60 \%$ of dry matter. Amongst the various classes of lipids involved, such as fatty acids, phospholipids and cholesterol, membrane of neurons are particularly enriched in fatty acids and complex lipids.

Cholesterol is the major component of cellular membranes. Its insertion between phospholipids forming the membrane bilayer-composed of the internal leaflet facing the cytoplasm and the external leaflet isolating the cell from the extracellular

\footnotetext{
*Correspondence: marie-claude.potier@upmc.fr
}

environment- ensures the structure and stability of the membrane. Cholesterol fluidifies the membrane, thus avoiding crystallization of fatty acids. It also decreases membrane permeability to hydrosoluble molecules. In the membranes, cholesterol is enriched in lipid rafts, which are essential nanometric domains involved in anchoring functional molecules such as membrane receptors (Simons and Toomre, 2000; Simons and Gerl, 2010). In neurons, cholesterol is highly enriched in myelin sheaths and allows the propagation of nerve impulses by saltatory mechanisms.

Alzheimer's disease (AD) is characterized by two abundant lesions, namely the extracellular amyloid plaques and the intracellular neurofibrillary tangles composed of hyperphosphorylated tau protein (Duyckaerts et al., 2009). Amyloid plaques are mainly composed of amyloid $\beta$ peptides 
(A $\beta$ ) produced by processing of the transmembrane Amyloid Precursor Protein APP by two sequential cleavages operated by the $\beta$ secretase BACE1 and the $\gamma$ secretase acting in the membrane bilayer (Selkoe and Hardy, 2016). The amyloidogenic APP processing takes place in the endosomal compartment after internalization of APP where the environmental $\mathrm{pH}$ is suitable for enzyme activities (De Strooper and Annaert, 2010).

A large body of evidence from genetic, neuropathological and biochemical studies suggest strong links between cholesterol and $\mathrm{AD}$, both in humans and in animal models of the disease (Cossec et al., 2010a).

\section{Genetic risk factors for AD involved in cholesterol homeostasis}

At least two genetic risk factors for $\mathrm{AD}$ are directly involved in cholesterol homeostasis (Lambert et al., 2013). The $\varepsilon 4$ allele of the apolipoprotein gene $A P O E$ is 2.7 fold more frequent in $\mathrm{AD}$ patients (36.7\%) as compared to the general population $(13.7 \%)$ while the frequency of the $\varepsilon 3$ allele is lower (59.4\%) in $\mathrm{AD}$ patients as compared to the general population (77.9\%) (Liu et al., 2013). Interestingly the $\varepsilon 2$ allele is more frequent $(8.4 \%)$ in the general population than in $\mathrm{AD}$ patients (3.9\%). Isoform specific amino acid differences lie at codons 112 and 158 with ApoE4 bearing two Arg, ApoE2 two Cys and ApoE3 a Cys at codon 112 and an Arg at codon 158. APOE $\varepsilon 4$ non-carriers have $\mathrm{AD}$ frequency of $20 \%$ with mean age of clinical onset at 84 while $A P O E \varepsilon 4$ heterozygous and homozygous are at $47 \%$ or $91 \%$, with mean age of onset of 76 or 68, respectively (Liu et al., 2013). Thus the $A P O E \& 4$ has a very significant effect both on frequency and age of onset of AD. Recently we found that in a cohort of 318 participants aged $70-85$ years with subjective memory complaints but unimpaired cognition and memory, $20 \%$ carried at least one $\varepsilon 4$ allele. When stratified according to their brain amyloid load assessed by positron emission tomography (PET), $38 \%$ were $\varepsilon 4$ among participants with $\mathrm{A} \beta$ deposition while the percentage dropped to $13 \%$ among participants with no $\mathrm{A} \beta$ deposition (Dubois et al., 2018). The $A P O E \& 4$ allele remains the highest genetic risk factor for $\mathrm{AD}$. Besides, genetic meta-analysis identified another risk factor involved in cholesterol homeostasis: the ATP binding cassette $\mathrm{ABC}$ transporter 7 ( $A B C A 7)$ gene. Intriguingly, $A B C A 7$ has one of the strongest association with amyloid deposition, exceeded only by APOE \&4 (Apostolova et al., 2018).

\section{Neuropathological studies showing increased cholesterol levels in the brain of AD patients}

A number of converging studies point towards an increase in cholesterol levels in human autopsy brains of AD patients, compared to age-matched unaffected controls. Evidence from biochemical studies suggested membrane-associated oxidative stress, resulting in perturbed cholesterol metabolism and increased cholesterol levels in the middle frontal gyrus of $\mathrm{AD}$ patients (Cutler et al., 2004). Of note, the same authors also demonstrated that the increase in membrane-associated cholesterol correlated with the severity of cognitive deficits (Cutler et al., 2004). An independent biochemical and histochemical study also showed that cholesterol levels are increased in the occipital cortices of AD patients (Xiong et al., 2008). A liquid chromatography coupled with mass spectrometry approach identified twice as much cholesterol in laser microdissected-A $\beta$ deposits from the isocortex of $A D$ patients compared to the adjacent neuropil (Panchal et al., 2010). Using time-of-flight secondary ion mass spectrometry imaging on cortical human sections revealed that the cholesterol signal was significantly higher in the cortical layers III and IV of AD samples compared to controls (Lazar et al., 2012).

\section{Biochemical evidence linking cholesterol, APP and its cleaving enzymes}

Cholesterol in the adult brain is produced by astrocytes that can either recapture it from the extracellular compartment or synthesize it using their specific enzyme machinery (Shobab et al., 2005). Synthetized free cholesterol then binds to APOE and is transported across the cell membrane via the ABCA1 transporter. Lipoparticles will bind to low density lipoprotein receptor-related protein 1 (LRP1) present at the neuronal membrane and be internalized in the endolysosomal compartment where APOE is degraded and free cholesterol released and relocated into cholesterol enriched membrane domains. APP, BACE1 and the $\gamma$ secretase protein complex are all localized in lipid rafts, suggesting a role of cholesterol in their processing activities (Bouillot et al., 1996; Cordy et al., 2003; Marquer et al., 2011). Indeed, an increase in cholesterol promoted $\gamma$ secretase activity and amyloid peptides production (Matsumura et al., 2014). Other studies demonstrated that the palmitoylation of APP regulates its distribution in lipid rafts and its amyloidogenic processing (Bhattacharyya et al., 2013). The palmitoylation of BACE1 also regulates its enrichment in lipid rafts and although it does not affect its steady-state processing activity, it controls the synaptic activity-induced production of $\mathrm{A} \beta$ (Vetrivel et al., 2009; Andrew et al., 2017).

\section{Structural evidence for a role of cholesterol on APP structure and dimerization at the membrane}

Although APP is a transmembrane protein that is present in cholesterol enriched domains, its sequence does not contain any cholesterol recognition amino acid consensus sequence (CRAC) or inverted consensus sequence CARC (Fantini et al., 2016). However, a binding site for cholesterol on APP Cterminal fragment $(\beta \mathrm{CTF})$ originating from the cleavage of APP by BACE1, has been predicted from structural studies using nuclear magnetic resonance (NMR) (Barrett et al., 2012). It was shown that the transmembrane and the juxtamembrane domains of $\beta \mathrm{CTF}$ are important for binding cholesterol, and molecular modelisation predicted that the transmembrane region of APP is a flexibly curved ahelix (Barrett et al., 2012). In addition, binding of cholesterol depends on the charge state of two amino acids (glutamic acid and aspartic acid at positions 22 and 23 of $A \beta$ sequence, respectively) present in the juxtamembrane domain of APP. At 
low $\mathrm{pH}$, these amino acids are neutral and bind cholesterol, while at higher $\mathrm{pH}$, they are negatively charged and inserted deeper in the membrane (Panahi et al., 2016).

A competition between binding of APP to cholesterol and its dimerization has been suggested (Song et al., 2013), as similar portions of APP are involved in both functions. Indeed, initial studies with $A \beta$ peptides showed that $A \beta 29-42$ is tilted and forms dimers in the membrane (Pillot et al., 1996). Moreover, dimerization of APP has been found to be due to the presence of GxxxG motifs in the transmembrane region (Kienlen-Campard et al., 2008). More precisely, the transmembrane motif $\mathrm{G}_{33} \mathrm{XXXG}_{37}$ and the juxtamembrane motif G25-SNK-G29 (numbering of $A \beta$ sequence) facilitate the dimerization of APP and cholesterol helps to stabilize this interface (Tang et al., 2014). Another study showed that APP dimerization is 5 times higher than with the CTF fragment, highlighting the important role of the APP extramembrane domain (Kaden et al., 2008; Ben Khalifa et al., 2012; Decock et al., 2015; Nierzwicki and Czub, 2015). Modelisation studies showed that the transmembrane helix of APP self-associates in left-handed parallel dimer while the juxtamembrane helix senses dimerization (Nadezhdin et al., 2012). Of note, the dimerization motif GxxxG is not sufficient for cholesterol binding and the juxtamembrane domain is required (Nierzwicki and Czub, 2015). Finally, a recent study showed that the transmembrane motif $\mathrm{V}_{39} \mathrm{XXA}_{42} \mathrm{~T}_{43} \mathrm{XXV}_{46} \mathrm{XXXV}_{50}$ is involved in the dimerization of $\mathrm{C} 99$ and binds lipid rafts (Sun et al., 2017).

\section{The role of cholesterol on APP endocytosis and processing}

Since APP is a transmembrane protein that can interact with cholesterol, and cholesterol levels are increased in AD, we studied the role of cholesterol on APP processing and amyloid pathology. We used methyßcyclodextrin (MBCD) to decrease cholesterol and MBCD-cholesterol complex to deliver cholesterol locally at the plasma membrane of living cells. Indeed, molecular dynamic studies have shown that MBCD can extract cholesterol from the plasma membrane (Lopez et al., 2011). We chose not to modulate the activity of cholesterol synthesis enzymes, such as 3-hydroxy-3-methylglutaryl-coenzyme A reductase (HMGCR), because of strong compensatory effects that take place when knocking down $H M G C R$ (data unpublished).

We demonstrated that, in HEK293 cells, membrane cholesterol levels control APP endocytosis and this effect is dependent on clathrin, the small GTPase Rab5 and dynamin2 (Cossec et al., 2010b). Dynamin2 is associated with clathrincoated pit maturation and fission of the membrane neck of coated pits. Rab5 regulates membrane fusion between early endosomes and their maturation through interaction with phosphatidylinositol 3-phosphate (PtdIns3P) and the early endosomal antigen 1 (EEA1). We found that the effect of cholesterol on APP endocytosis was inhibited by either transfecting dominant negative mutants of Rab5 or dynamin2 that cannot be activated, or by treating cells with siRNA against clathrin (Cossec et al., 2010b).

Using fluorescence activated cell sorting (FACS), we confirmed that APP endocytosis is promoted by cholesterol

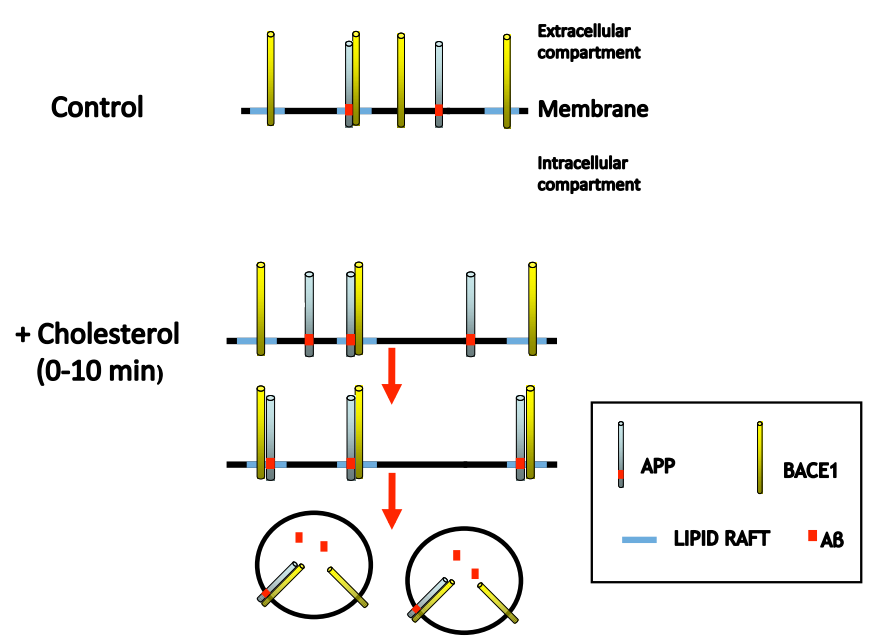

Fig. 1. Effects of artificial increase of cholesterol at the cellular plasma membrane.

In the control condition, APP and BACE1 are present inside and outside lipid rafts at the plasma membrane. Raising cholesterol at the plasma membrane induces: i) relocalization of APP and BACE1 in lipid rafts and their rapid internalization assessed by Fluorescence correlation Spectroscopy in enlarged and clustered endosomes (Marquer et al., 2011; Marquer et al., 2014); ii) increase of APP and BACE1 proximity assessed by Total Internal Reflection Fluorescence-Fluorescence Lifetime Imaging-Förster Resonance Energy Transfer (TIRF-FLIM-FRET) microscopy (Marquer et al., 2011); iii) increase of production of A peptides (Cossec et al., 2010a; Cossec et al., 2010b; Marquer et al., 2014).

increase in a dose-dependent manner (Cossec et al., 2010b). To explore the specificity of the effect of cholesterol levels on internalization, we tested it on the cannabinoid 1 (CB1) and the transferrin receptors, and found that an increase of cholesterol at the plasma membrane only slightly promoted their internalization. In contrast, APP endocytosis was much more sensitive to the levels of cholesterol. In addition, we found that cholesterol treatment increased APP internalization in endosomes that appeared larger than in untreated cells. There was a significant increase of $A \beta 40$ and $A \beta 42$ secretion in HEK293 cells transfected with APP bearing the Swedish mutations and treated with MBCD-cholesterol complex. This effect was dependent on the extent of the cholesterol increase at the plasma membrane and on APP internalization, as demonstrated by clathrin knock-down experiments (Cossec et al., 2010b). A similar effect of cholesterol on endogenous $A \beta 42$ secretion was reproduced in primary rat hippocampal neurons, although, in this case, $A \beta 40$ was unchanged and $A \beta 38$ was decreased (Marquer et al., 2014).

Increase of $A \beta$ secretion following cholesterol treatment could be due to either an effect on BACE1 activity or to a facilitation of APP-BACE1 proximity. After showing that induced cholesterol increase at the plasma membrane had no effect on BACE1 catalytic activity, we thought to study the interaction of APP and BACE1 at the plasma membrane of primary neurons in culture (Marquer et al., 2011). Rat hippocampal neurons were transfected with APP-mCherry and BACE1-GFP constructs and imaged using Fluorescence Lifetime Imaging Microscopy (FLIM). When the distance 
between the two proteins (BACE1-GFP donor and APPmCherry acceptor) is below $10 \mathrm{~nm}$, Förster Resonance Energy Transfer (FRET) can occur and the fluorescence lifetime of the donor decreases. By Total Internal Reflection Fluorescence (TIRF) imaging, we could demonstrate that increase of cholesterol favors APP-BACE1 proximity at the plasma membrane. We then wondered whether this happened in specific regions of the plasma membrane enriched in cholesterol (lipid rafts). We used Fluorescence Correlation Spectroscopy (FCS) to measure the diffusion coefficients of APP-YFP molecules (Marquer et al., 2012). We showed that about $7 \mathrm{~min}$ after cholesterol treatment, APP-YFP relocalized to lipid rafts where diffusion time is higher as compared to non-raft portions of the plasma membrane (Marquer et al., 2011). This peak was followed by an extinction of fluorescence signal attributed to the internalization of APP-YFP, since this fluorescence decrease was inhibited by dynasore treatment and corresponded to the time of internalization through the clathrin-dependant pathway. Taken together, our microscopy studies strongly suggest that, in normal conditions, APP and BACE1 are distributed inside and outside of lipid rafts. However, less than $10 \mathrm{~min}$ after treatment with MBCDcholesterol, APP and BACE1 are relocalized in lipid rafts that have either higher levels of cholesterol or expand their size with similar levels of cholesterol per surface unit. This event is rapidly followed by internalization in early endosomes that have greater size (Fig. 1).

Altogether, we show that modulating cholesterol levels to the extent of what is observed in human AD autopsy brains $(+30 \%)$ results in increased APP and BACE1 proximity and in their endocytosis in enlarged early endosomes, leading to the overproduction of $A \beta$ peptides. This enlarged endosome phenotype is reminiscent of what has been described in pyramidal cells from postmortem human brain.

\section{Increasing neuronal cholesterol levels recapitulates $A D$ phenotypes}

We also explored whether, in addition to its effect on $A \beta$ levels, increasing cholesterol could also lead to other cellular phenotypes reminiscent of $\mathrm{AD}$. As previously mentioned, one of the earliest phenotypes of human $\mathrm{AD}$ is the enlargement of early endosomes (Cataldo et al., 2000). We found that treatment of primary rat hippocampal neurons with MBCDcholesterol to reach a $30 \%$ increase, induced a change in the endosomal compartment with an increase in the mean volume of early endosomes, as assessed by confocal microscopy (Marquer et al., 2014). Using transmission electron microscopy, we found that not only the surface of early endosomes was increased but they also formed clusters. Another key feature of $\mathrm{AD}$ dysfunction is axonal transport deficits (Dai et al., 2002). We thus analyzed axonal transport of APP. Hippocampal neurons were transfected with APP-mCherry and treated with MBCD-cholesterol for 20-35 min and APP-mCherry containing vesicles were followed using live imaging. We found a very significant decrease of APP anterograde transport after cholesterol increase, while retrograde transport remained unchanged (Marquer et al., 2014). Finally and in order to find out whether cholesterol treated neurons had a similar transcripts signature as compared to neurons from postmortem human brain samples, we compared the transcriptome of treated neurons to the ones from brain samples at different Braak stages of AD. Hierarchical clustering analysis highlighted that gene expression profile of cholesterol-treated neurons were similar to early AD stages, suggesting that cholesterol increase could be an early phenotype during the course of the disease, either occurring as a primary or secondary event (Marquer et al., 2014). Among the genes differentially expressed following cholesterol increase, Gene Ontology category enrichment analysis revealed that cholesterol pathway, response to lipid, sterol biosynthetic pathways were the most enriched categories.

Thus, increase in neuronal membrane cholesterol promotes APP processing and endocytosis, enlargement of early endosomes and axonal transport abnormalities, inducing gene expression modifications similar to the ones detected in early stages of AD. Changes in membrane cholesterol linked with age could thus be a starting event triggering sporadic AD.

This postulate based on evidence collected in neuronal cultures is supported by in vivo studies. For example, inhibiting CYP46A1, the enzyme that hydroxylates cholesterol at position 24, allowing its passage through the blood brain barrier and the decrease of cholesterol levels in the brain, worsens AD phenotypes. Specifically, CYP46A1 inhibition leads to brain cholesterol accumulation, endoplasmic reticulum stress, increase of $\beta C T F$ and $A \beta$ production, tau hyperphosphorylation and hippocampal atrophy linked to neuronal death, supporting that high neuronal cholesterol could induce or accelerate the course of sporadic AD (Djelti et al., 2015). In addition, crossing APP/PS1 mice with mice overexpressing sterol regulatory element-binding protein-2 (SREBP2) induced mitochondrial cholesterol accumulation, oxidative damage and $\mathrm{A} \beta$ accumulation as well as, very interestingly, tau phosphorylation and neurofibrillary tangle formation that had never been detected before in any mouse model reproducing the amyloid pathology (Barbero-Camps et al., 2013).

\section{Towards decreasing neuronal membrane cholesterol to delay/counteract sporadic AD}

Specifically decreasing neuronal cholesterol thus appears like a promising strategy to either delay or counteract the development of sporadic AD. Several studies in mouse models convincingly support this hypothesis. Injection of adenoviral constructs expressing CYP46A1 in mouse models of $\mathrm{AD}$ reproducing either the amyloid or the tau pathologies improves cognitive function and reduces amyloid pathology as well as tau hyperphosphorylation (Hudry et al., 2010; Burlot et al., 2015). CYP46A1 knock-out mice have severe memory deficits (Kotti et al., 2006) while older transgenic mice overexpressing CYP46A1 show improved cognitive performance (Maioli et al., 2013). In addition, treatment of $\mathrm{AD}$ mice overexpressing APP with the Swedish mutations with hydroxypropyl$\beta$-cyclodextrin reduced the number of amyloid plaques, increased the expression of genes involved in cholesterol transport and $\mathrm{A} \beta$ clearance, and improved spatial learning and memory deficits (Yao and Papadopoulos, 2002), thus showing clear neuroprotective effects. 
Finally replacing membrane cholesterol with stigmasterol, a plant sterol crossing the blood brain barrier, decreased APP processing and the production of $A \beta$ peptides. Mice under stigmasterol-rich diet had decreased amyloidogenic APP processing (Burg et al., 2013). On the contrary, high cholesterol diet in a transgenic mouse model expressing Swedish and London mutations disturbs the integrity of the $\mathrm{BBB}$, increases $\mathrm{A} \beta$ in the brains and decreases $A \beta$ in the plasma of these mice (Loffler et al., 2016).

In total, results from these studies, using various strategies to decrease neuronal cholesterol, converge towards the conclusion that lowering cholesterol levels in the brain is indeed beneficial and alleviates an array of $\mathrm{AD}$ phenotypes.

\section{Conclusions}

Since the identification of the cholesterol transporter APOE $\varepsilon 4$ haplotype as the highest genetic risk factor for AD (Mayeux et al., 1993), much has been done to understand the role of cholesterol metabolism in the pathogenesis of AD (Allinquant et al., 2014). We deliberately omitted all the work performed with statins, since most of the clinical and molecular studies have not been conclusive so far (Yasar and Whitmer, 2018). A significant body of work points to a deleterious role of neuronal cholesterol in the development of sporadic AD. Amongst these, molecular studies and new microscopies allowing the analysis of sub compartments of the plasma membrane provided definitive evidence of this role. Measuring in situ cholesterol levels in postmortem human brain samples remains a challenge. Without any selective antibodies, we are left with very few markers that remain extremely difficult to utilize. Some therapies have been tested preclinically in mouse models of AD but much remains to be done in clinical research to validate possible targets and food supplements in order to delay or counteract sporadic AD. Finally, a more precise molecular analysis of the APP cholesterol binding site and the important yet misunderstood interplay with APOE genotype might bring new insights into the field and pave the way to unprecedented therapeutic avenues.

\section{Disclosure}

The authors disclose any conflict of interest.

Acknowledgements. The authors wish to thank Institut de Recherche Servier and the program "Investissements d'avenir" ANR-10-IAIHU-06 for their financial support. LH had a fellowship from Institut de Recherche Servier.

\section{References}

Allinquant B, Clamagirand C, Potier MC. 2014. Role of cholesterol metabolism in the pathogenesis of Alzheimer's disease. Curr Opin Clin Nutr Metab Care 17: 319-323.

Andrew RJ, Fernandez CG, Stanley M, et al. 2017. Lack of BACE1 Spalmitoylation reduces amyloid burden and mitigates memory deficits in transgenic mouse models of Alzheimer's disease. Proc Natl Acad Sci USA 114: E9665-E9674.
Apostolova LG, Risacher SL, Duran T, et al. 2018. Associations of the top 20 Alzheimer disease risk variants with brain amyloidosis. JAMA Neurol 75: 328-341.

Barbero-Camps E, Fernandez A, Martinez L, Fernandez-Checa JC, Colell A. 2013. APP/PS1 mice overexpressing SREBP-2 exhibit combined Abeta accumulation and tau pathology underlying Alzheimer's disease. Hum Mol Genet 22: 3460-3476.

Barrett PJ, Song Y, Van Horn WD, et al. 2012. The amyloid precursor protein has a flexible transmembrane domain and binds cholesterol. Science 336: 1168-1171.

Ben Khalifa N, Tyteca D, Marinangeli C, et al. 2012. Structural features of the KPI domain control APP dimerization, trafficking, and processing. FASEB $J$ 26: 855-867.

Bhattacharyya R, Barren C, Kovacs DM. 2013. Palmitoylation of amyloid precursor protein regulates amyloidogenic processing in lipid rafts. J Neurosci 33: 11169-11183.

Bouillot C, Prochiantz A, Rougon G, Allinquant B. 1996. Axonal amyloid precursor protein expressed by neurons in vitro is present in a membrane fraction with caveolae-like properties. $\mathrm{J} \mathrm{Biol} \mathrm{Chem}$ 271: 7640-7644.

Burg VK, Grimm HS, Rothhaar TL, et al. 2013. Plant sterols the better cholesterol in Alzheimer's disease? A mechanistical study. J Neurosci 33: 16072-16087.

Burlot MA, Braudeau J, Michaelsen-Preusse K, et al. 2015. Cholesterol 24-hydroxylase defect is implicated in memory impairments associated with Alzheimer-like Tau pathology. Hum Mol Genet 24: 5965-5976.

Cataldo AM, Peterhoff CM, Troncoso JC, Gomez-Isla T, Hyman BT, Nixon RA. 2000. Endocytic pathway abnormalities precede amyloid beta deposition in sporadic Alzheimer's disease and Down syndrome: differential effects of APOE genotype and presenilin mutations. Am J Pathol 157: 277-286.

Cordy JM, Hussain I, Dingwall C, Hooper NM, Turner AJ. 2003. Exclusively targeting beta-secretase to lipid rafts by GPI-anchor addition up-regulates beta-site processing of the amyloid precursor protein. Proc Natl Acad Sci USA 100: 11735-11740.

Cossec JC, Marquer C, Panchal M, Lazar AN, Duyckaerts C, Potier MC. 2010a. Cholesterol changes in Alzheimer's disease: Methods of analysis and impact on the formation of enlarged endosomes. Biochim Biophys Acta 1801: 839-845.

Cossec JC, Simon A, Marquer C, et al. 2010b. Clathrin-dependent APP endocytosis and Abeta secretion are highly sensitive to the level of plasma membrane cholesterol. Biochim Biophys Acta 1801: 846-852.

Cutler RG, Kelly J, Storie K, et al. 2004. Involvement of oxidative stress-induced abnormalities in ceramide and cholesterol metabolism in brain aging and Alzheimer's disease. Proc Natl Acad Sci USA 101: 2070-2075.

Dai J, Buijs RM, Kamphorst W, Swaab DF. 2002. Impaired axonal transport of cortical neurons in Alzheimer's disease is associated with neuropathological changes. Brain Res 948: 138-144.

De Strooper B, Annaert W. 2010. Novel research horizons for presenilins and gamma-secretases in cell biology and disease. Annu Rev Cell Dev Biol 26: 235-260.

Decock M, El Haylani L, Stanga S, et al. 2015. Analysis by a highly sensitive split luciferase assay of the regions involved in APP dimerization and its impact on processing. FEBS Open Bio 5: 763-773.

Djelti F, Braudeau J, Hudry E, et al. 2015. CYP46A1 inhibition, brain cholesterol accumulation and neurodegeneration pave the way for Alzheimer's disease. Brain 138: 2383-2398.

Dubois B, Epelbaum S, Nyasse F, et al. 2018. Cognitive and neuroimaging features and brain beta-amyloidosis in individuals 
at risk of Alzheimer's disease (INSIGHT-preAD): a longitudinal observational study. Lancet neurology 17: 335-346.

Duyckaerts C, Delatour B, Potier MC. 2009. Classification and basic pathology of Alzheimer disease. Acta Neuropathol 118: 5-36.

Fantini J, Di Scala C, Evans LS, Williamson PT, Barrantes FJ. 2016. A mirror code for protein-cholesterol interactions in the two leaflets of biological membranes. Sci Rep 6: 21907.

Hudry E, Van Dam D, Kulik W, et al. 2010. Adeno-associated virus gene therapy with cholesterol 24-hydroxylase reduces the amyloid pathology before or after the onset of amyloid plaques in mouse models of Alzheimer's disease. Mol Ther 18: 44-53.

Kaden D, Munter LM, Joshi M, et al. 2008. Homophilic interactions of the amyloid precursor protein (APP) ectodomain are regulated by the loop region and affect \{beta\}-secretase cleavage of APP. $J$ Biol Chem 283: 7271-7279.

Kienlen-Campard P, Tasiaux B, Van Hees J, et al. 2008. Amyloidogenic processing but not amyloid precursor protein (APP) intracellular C-terminal domain production requires a precisely oriented APP dimer assembled by transmembrane GXXXG motifs. J Biol Chem 283: 7733-7744.

Kotti TJ, Ramirez DM, Pfeiffer BE, Huber KM, Russell DW. 2006. Brain cholesterol turnover required for geranylgeraniol production and learning in mice. Proc Natl Acad Sci USA 103: 3869-3874.

Lambert JC, Ibrahim-Verbaas CA, Harold D, et al. 2013. Metaanalysis of 74,046 individuals identifies 11 new susceptibility loci for Alzheimer's disease. Nat Genet 45: 1452-1458.

Lazar AN, Bich C, Panchal M, et al. 2012. Time-of-flight secondary ion mass spectrometry (TOF-SIMS) imaging reveals cholesterol overload in the cerebral cortex of Alzheimer disease patients. Acta Neuropathol 125: 133-144.

Liu CC, Kanekiyo T, Xu H, Bu G. 2013. Apolipoprotein E and Alzheimer disease: risk, mechanisms and therapy. Nat Rev Neurol 9: 106-118.

Loffler T, Flunkert S, Temmel M, Hutter-Paier B. 2016. Decreased plasma abeta in hyperlipidemic APPSL transgenic mice is associated with BBB dysfunction. Front Neurosci 10: 232.

Lopez CA, de Vries AH, Marrink SJ. 2011. Molecular mechanism of cyclodextrin mediated cholesterol extraction. PLoS comput biol 7: e1002020.

Maioli S, Bavner A, Ali Z, et al. 2013. Is it possible to improve memory function by upregulation of the cholesterol 24Shydroxylase (CYP46A1) in the brain? PLoS One 8: e68534.

Marquer C, Devauges V, Cossec JC, et al. 2011. Local cholesterol increase triggers amyloid precursor protein-Bacel clustering in lipid rafts and rapid endocytosis. FASEB $J$ 25: 1295-1305.

Marquer C, Laine J, Dauphinot L, et al. 2014. Increasing membrane cholesterol of neurons in culture recapitulates Alzheimer's disease early phenotypes. Mol neurodegener 9: 60.

Marquer C, Leveque-Fort S, Potier MC. 2012. Determination of lipid raft partitioning of fluorescently-tagged probes in living cells by fluorescence correlation spectroscopy (FCS). J Vis Exp 62: e3513.

Matsumura N, Takami M, Okochi M, et al. 2014. Gamma-Secretase associated with lipid rafts: multiple interactive pathways in the stepwise processing of beta-carboxyl-terminal fragment. $J$ Biol Chem 289: 5109-5121.

Mayeux R, Stern Y, Ottman R, et al. 1993. The apolipoprotein epsilon 4 allele in patients with Alzheimer's disease. Ann Neurol 34: $752-754$.

Nadezhdin KD, Bocharova OV, Bocharov EV, Arseniev AS. 2012. Dimeric structure of transmembrane domain of amyloid precursor protein in micellar environment. FEBS Lett 586: 1687-1692.

Nierzwicki L, Czub J. 2015. Specific binding of cholesterol to the amyloid precursor protein: structure of the complex and driving forces characterized in molecular detail. J Phys Chem Lett 6: 784-790.

Panahi A, Bandara A, Pantelopulos GA, Dominguez L, Straub, JE. 2016. Specific binding of cholesterol to C99 domain of amyloid precursor protein depends critically on charge state of protein. $J$ Phys Chem Lett 7: 3535-3541.

Panchal M, Loeper J, Cossec JC, et al. 2010. Enrichment of cholesterol in microdissected Alzheimer's disease senile plaques as assessed by mass spectrometry. J Lipid Res 51: 598-605.

Pillot T, Goethals M, Vanloo B, et al. 1996. Fusogenic properties of the C-terminal domain of the Alzheimer beta-amyloid peptide. $J$ Biol Chem 271: 28757-28765.

Selkoe DJ, Hardy J. 2016. The amyloid hypothesis of Alzheimer's disease at 25 years. EMBO mol med 8: 595-608.

Shobab LA, Hsiung GY, Feldman HH. 2005. Cholesterol in Alzheimer's disease. Lancet neurology 4: 841-852.

Simons K, Gerl MJ. 2010. Revitalizing membrane rafts: new tools and insights. Nat Rev Mol Cell Biol 11: 688-699.

Simons K, Toomre D. 2000. Lipid rafts and signal transduction. Nat Rev Mol Cell Biol 1: 31-39.

Song Y, Hustedt EJ, Brandon S, Sanders CR. 2013. Competition between homodimerization and cholesterol binding to the C99 domain of the amyloid precursor protein. Biochemistry 52: 5051-5064.

Sun F, Chen L, Wei P, et al. 2017. Dimerization and structural stability of amyloid precursor proteins affected by the membrane microenvironments. J Chem Inf Model 57: 1375-1387.

Tang TC, Hu Y, Kienlen-Campard P, et al. 2014. Conformational changes induced by the A21G Flemish mutation in the amyloid precursor protein lead to increased Abeta production. Structure 22: 387-396.

Vetrivel KS, Meckler X, Chen Y, et al. 2009. Alzheimer disease Abeta production in the absence of S-palmitoylation-dependent targeting of BACE1 to lipid rafts. $J$ Biol Chem 284: 3793-3803.

Xiong H, Callaghan D, Jones A, et al. 2008. Cholesterol retention in Alzheimer's brain is responsible for high beta- and gammasecretase activities and A beta production. Neurobiol Dis 29: 422-437.

Yao ZX, Papadopoulos V. 2002. Function of beta-amyloid in cholesterol transport: a lead to neurotoxicity. Faseb $J$ 16: 16771679.

Yasar S, Whitmer R. 2018. Statin use and risk of Alzheimer disease: A new view on an old relationship. Neurology 90: 103-104.

Cite this article as: Potier M-C, Hanbouch L, Marquer C. 2018. Cholesterol and ApoE in Alzheimer's disease. OCL 25(4): D407. 